\title{
Simple experimental technique for measuring lifetimes of low-intensity monoexponential fluorescence signals
}

Fernando Moreno, R. Lopez, P. Valle Herrero, Jose Saiz, Francisco Gonzalez

Fernando Gracia Moreno, R. J. Lopez, P. J. Valle Herrero, Jose M. Saiz, Francisco Gonzalez, "Simple experimental technique for measuring lifetimes of low-intensity monoexponential fluorescence signals," Proc. SPIE 1603, Education in Optics, (1 March 1992); doi: 10.1117/12.57883 


\title{
A simple experimental technique for measuring lifetimes of low intensity monoexponential fluorescence signals
}

\author{
F. Moreno, R.J. López, P.J. Valle, J.M. Saiz and F. González \\ Departamento de Física Aplicada. Universidad de Cantabria \\ 39005 Santander (Spain)
}

\begin{abstract}
$\underline{\text { ABSTRACT }}$
A simple experimental technique for measuring the lifetime of long-lived excited levels for low intensity monoexponential fluorescence signals is presented. It is based on the measurement of the imaginary part of the Fourier transform of the probability density function of the time of arrival of the first photon after the excitation. Owing to its theoretical and practical contents (photon counting techniques, lifetime measurement techniques), this experimental procedure may constitute a suitable practice for undergraduate students in Optics and Solid State Physics.
\end{abstract}

\section{INTRODUCTION}

Based on our recent studies about ${ }^{1-3}$ photon statistics of deterministic signals of low intensity, we present in this work a simple experimental procedure for determining the lifetime of long-lived excited levels when the intensity of fluorescence is low (less than one photoelectron per lifetime) and the decay is represented by only one exponential. This experiment can constitute a suitable practice for undergraduate students in Optics and Solid State Physics, since not only information about the internal constitution of some solid compounds can be obtained but introduces new experiments with photoncounting techniques.

As has been established previously for monoexponentially decaying signals, the imaginary part of the Fourier transform of the probability distribution function (IFPDF) of the time of arrival of the first fluorescence photon after the excitation shows a peak centered at the frequency $\omega_{0}=\tau_{0}{ }^{-1}, \tau_{0}$ being the lifetime of the excited level. The measurement of the IFPDF and its fitting to a simple curve (a dispersion curve in our case) give the required parameter $\tau_{0}$. The experimental set-up is very simple: a modulated laser beam tuned to the appropriate frequency, a photon counting detection system, a timeinterval counter and a desk computer to collect the time intervals and to perform the calculation of the IFPDF. By using standard fitting techniques the parameter $\tau_{0}$ and its corresponding error can be easily obtained by repeating the experiment a fixed number of times.

The proposed experiment may be complemented by comparing these results with those obtained through the conventional single photon decay spectroscopy (SPDS) technique for the same experimental conditions in order to show the advantages and disadvantages of both methods. 


\section{THEORY}

The intensity emitted by fluorescence depends on time which, in the case of single exponential decay can be given by

$$
I(t)=I_{0} \exp \left(-t / \tau_{0}\right),
$$

where $\tau_{0}$ is the decay constant.

The probability distribution function of the time of arrival of the first photoelectron, $P_{f}(t)$, is obtained from $\mathrm{I}(\mathrm{t})$ and is given by 4

$$
P_{f}(t)=I(t) \exp \left(-\int_{0}^{t} I\left(t^{\prime}\right) d t^{\prime}\right)
$$

By substituting expression (1) in (2), the complete theoretical expression can be obtained

$$
P_{f}(t)=I_{0} \exp \left(-t / \tau_{0}\right) \exp \left(-I_{0} \tau_{0}\left[1-\exp \left(-t / \tau_{0}\right)\right]\right)
$$

The SPDS technique is used in experimental situations in which $I_{0} \tau_{0}<<1$ is verified. In this case, the simplified equation frequently used is

$$
P_{f}(t) \cong I(t)=I_{0} \exp \left(-t / \tau_{0}\right)
$$

As indicated in the introduction, the method proposed in previous papers 1,2 consisted of directly measuring the Laplace Transform of the function $P_{f}(t)$ without having to construct this probability distribution first: that is, using a time interval counter, the $t_{i}$ were measured and the experimental estimator $\hat{Q}_{L}(s)$ was constructed being defined as

$$
\hat{\mathrm{Q}}_{\mathrm{L}}(\mathrm{s})=\left(1 / \mathrm{N}_{\mathrm{c}}\right) \sum_{i=1}^{\mathrm{N}} \exp \left(-s \mathrm{t}_{\mathrm{i}}\right)
$$

$N_{c}$ being the number of excitation cycles and $N$ the number of $t_{i}$ which were inferior to five times the decay time expected in the experiment 5 .

The theoretical value of the function $Q_{L}(s)$ was readily found from

$$
Q_{L}(s)=<\exp (-s t)>=\int_{0}^{\infty} P_{f}(t) \exp (-s t) d t .
$$

For low light levels, the theoretical expression for $\mathrm{Q}_{\mathrm{L}}(\mathrm{s})$ takes a simple form

$$
\mathrm{Q}_{\mathrm{L}}(\mathrm{s})=\mathrm{I}_{0} \tau_{0} \exp \left(-\mathrm{I}_{0} \tau_{0}\right) /\left(1+\mathrm{s} \tau_{0}\right)
$$


which is an ever decreasing function for $\mathrm{s}>0$.

Moreover, if expression (6) is developed in powers of $t$, we obtain

$$
\mathrm{Q}_{\mathrm{L}}(\mathrm{s})=\sum_{\mathrm{k}=0}^{\mathrm{N}}(-\mathrm{s})^{\mathrm{k}}<\mathrm{t} \mathrm{k}>/ \mathrm{k} !
$$

an expression which reveals the relationship between $Q_{L}(s)$ and the factorial moments of $P_{f}(t)$.

In this paper we are interested in the Fourier Transform of $P_{f}(t)$

$$
\begin{gathered}
T_{F}(f)=\int_{-\infty}^{\infty} \exp (-i 2 \pi f t) P_{f}(t) d t= \\
\int_{-\infty}^{\infty} \cos (2 \pi f t) P_{f}(t) d t-i \int_{-\infty}^{\infty} \sin (2 \pi f t) P_{f}(t) d t= \\
<\cos (2 \pi f t)>-i<\sin (2 \pi f t)>=T_{\cos }(f)-i T_{\sin }(f) .
\end{gathered}
$$

By introducing into these expressions the signals $P_{f}(t)$ for low light intensity levels (Eq.(4)) we obtain

$$
\begin{gathered}
\left|T_{F}(f)\right|=I_{0} /\left[\left(1 / \tau_{0}\right)^{2}+(2 \pi f)^{2}\right]^{1 / 2}, \\
T_{\cos }(f)=<\cos (2 \pi f t)>=\left(I_{0} / \tau_{0}\right) /\left[\left(1 / \tau_{0}\right)^{2}+(2 \pi f)^{2}\right], \\
T_{\sin }(f)=<\sin (2 \pi f t)>=2 \pi f I_{0} /\left[\left(1 / \tau_{0}\right)^{2}+(2 \pi f)^{2}\right] .
\end{gathered}
$$

The graphs corresponding to formulae (10) for positive values of $f$ are given in Fig. 1. As can be observed, while $\left|T_{F}(f)\right|$ and $T_{\text {cos }}(f)$ are ever decreasing functions, $T_{\sin }(f)$ (IFPDF) shows a maximum for a value of $f_{m}=1 /\left(2 \pi \tau_{0}\right)$, where the function takes the value $I_{0} \tau_{0} / 2$ and with a fullwidth of $\Delta f=2 \sqrt{3} f_{m}$ as is readily deduced from (10c). The position of this maximum, therefore, allows the decay time value to be identified easily.

The experimental estimator for $T_{\sin }(f)$ is given by

$$
\hat{\mathrm{T}}_{\sin }(\mathrm{f})=\left(1 / \mathrm{N}_{\mathrm{c}}\right) \sum_{\mathrm{i}=1}^{\mathrm{N}} \sin \left(2 \pi \mathrm{ft}_{\mathrm{i}}\right)
$$

where the symbols have the same significance as in expression (5).

Moreover, by performing for $\mathrm{T}_{\sin }(\mathrm{f})$ the serial development analogous to that indicated for $\mathrm{Q}_{\mathrm{L}}(\mathrm{s})$ in expression (8), we obtain

$$
T_{\sin }(f)=\int_{-\infty}^{\infty} \sin (2 \pi f t) P_{f}(t) d t=
$$




$$
\sum_{k=0}^{\infty}(-1)^{k}(2 \pi f)^{2 k+1}<t^{2 k+1}>/(2 k+1) ! .
$$

Thus, $T_{\sin }(f)$ only contains information on the odd-numbered terms $\langle t\rangle,\left\langle t^{3}\right\rangle$, etc., while $Q_{L}(s)$ includes all terms. This disadvantage may be balanced by the presence of well defined maxima in the signal for $T_{\sin }(f)$.

\section{NUMERICAL SIMULATION OF THE EXPERIMENT}

Before the performance of the experimental analysis, a computer simulation is carried out in order to show how the simulation techniques work and how they can be applied to a real experiment. ${ }^{6}$ Two experimental situations for very low intensity fluorescence signals are simulated in order to compare the conventional multichannel technique with the proposed method. In both cases $\alpha$ is kept to 0.01 and the number of excitation cycles, $\mathrm{N}_{\mathrm{c}}$, chosen $10^{4}$ and $10^{5}$. A typical value of $\tau_{0}=1 \mathrm{msec}$ is selected. For this value of $\alpha$ and from $\mathrm{N}_{\mathrm{c}}=10^{4}$, only 100 photoelectrons are expected to be detected. If the interval $\left[0, t_{\max }=5 \tau_{0}\right]$ is divided into a minimum of 500 channels in order to construct $P_{f}(t)$ which fits expression (4), it is easy to understand that the 100 photoelectrons will be shared among the channels in such a way that will make any kind of fit impossible. Although in this case we do not give the graph for $P_{f}(t)$ for obvious reasons, we have given in Fig. 2 the values of $T_{\sin }(f)$ for this truly extreme case. It is surprising how, in spite of the sparse information, a maximum for a reasonably approximate value for $\tau_{0}$ appears.

In more realistic case, we have simulated an experiment with 105 measurement cycles maintaining the value of $\alpha=0.01$. In Fig. 3 we give the $P_{f}(t)$ with the corresponding straight line fit after having eliminated the channels in which no photoelectron existed. The great fluctuation existing can be seen which leads to a regression coefficient in the fit to a straight line in the order of 0.56 , which indicates the difficulty in distinguishing behaviour patterns different from that of the monoexponential. In Fig. 4 we show the graph for $T_{\sin }(f)$ when the same $t_{i}$ as in Fig. 3 were used. The maximum clearly stands out and indicates a single exponential decay behaviour in the signal and the decay time value.

\section{EXPERIMENT}

Next an experiment was carried out to measure the decay constant for a TMMC crystal at room temperature. This sample has been sufficiently studied by other authors 2 for the results obtained by measuring $T_{\sin }(f)$.

Our experimental setup, schematically shown in Fig. 5, consists of an $\mathrm{Ar}^{+}$laser (SpectraPhysics 2020-03) as the source of excitation, an acousto-optic light modulator (NRC N33-D), which admits pulses with a width of several $\mu$ sec. and is governed by a signal from a pulse generator (HP8005B). 
The photon-counter detection chain consists of an RCA cooled photomultiplier (C31034) and a amplifier-discriminator (PAR 1120). The signal can be treated by means of a correlator (Malvern K7025) operating in multichannel mode or by measuring the time intervals between the end of luminous excitation and detection of the first photoelectron with a photon-counter (HP-5308A) whose data are transferred to a HP-300 computer.

Owing to the emission and absorption spectra of the TMMC crystal, the sample was excited with $\lambda=476.5 \mathrm{~nm}$ and the light emitted was observed through an interference filter centered in 650 $\mathrm{nm}$.

For high intensities the multichannel technique gives the decay curve shown in Fig. 6 and from its fit we get $\tau_{0}=841.5 \mu \mathrm{sec}$. which agrees very well with other authors 2 .

For low intensities $\left(I_{0} \tau_{0}=0.4\right)$ we began with the technique of measuring $P_{f}(t)$ (SPDS technique). The results for $10^{4}$ excitation cycles are shown in Fig. 7. As with the computer simulation, fluctuations due to low signal-to-noise ratio for each channel can be seen. The straight line fit of $P_{f}(t)$ has a regression coefficient of 0.76 which shows the difficulty in discriminating the existence of deviations from the behaviour of a single exponential.

Using the same $t_{i}$ we constructed $T_{\text {sin }}(f)$ according to definition (11) and obtained the points in Fig. 8. Since the curve for $T_{\sin }(f)$ shows a maximum for $2 \pi f=1 / \tau_{0}\left(\approx 10^{3}\right.$ in our case), the $f$ values were chosen so that they covered approximately two orders of magnitude around the estimated value $\left(10^{2} \leq 2 \pi \mathrm{f} \leq 10^{4}\right)$. Higher values contain no significant information about the position of the maximum and show fluctuations which reduce the goodness of the fit. In Fig. 8 we show the best theoretical fitted function (continuous line) to the experimental points. Here, it is important to point out that the theoretical function (continuous line in Fig. 8) used in the fitting procedure is not given by Eq. (10c) but by a modified version of this. This is due to the fact that the experimental excitation cycle is not infinite but is given by the period of the excitation signal, $\mathrm{T}_{\max }$. If this is taken into account, the corrected version of Eq. (10c) is

$$
\left[T_{\sin }(f)\right]_{c}=T_{\sin }(f)-\int_{T_{\max }}^{\infty} \sin (2 \pi f t) P_{f}(t) d t,
$$

where the last term of the right hand side can be easily calculated. On the other hand Eq. (13) explains the appearance in the experimental points of the small shoulder close to the main peak. This is important since this shoulder could be interpreted erroneously as if another decay constant were present in the fluorescence signal.

Ten series of $10^{4}$ cycles were measured in the same conditions and the results of the fit for both techniques are given in Table 1 . The error in the determination of $\tau_{0}$ for the ten series was about $2.6 \%$ for the $\mathrm{T}_{\sin }(\mathrm{f})$ method.

The values of $\tau_{0}$ deduced from the measurement of $P_{f}(t)$ show a good average but the regression coefficient and the value of $\chi v^{2}$ prevents (see ref. 7) the appreciation of deviations from the assumed behaviour when the luminescence of an unknown sample is studied. 


\section{ACKNOWLEDGEMENTS}

This work has been supported by the Ministry of Education and Science of Spain (CICYT, Project PB86-0504). The authors want to express their gratitude for this financial support.

\section{REFERENCES}

1. F. Moreno, R.J. Lopez, "Lifetime measurement by using the Laplace Transform in Single-PhotonDecay spectroscopy", Appl. Spect., 41, 1307 (1987)

2. F. González, R.J. Lopez, F. Moreno, "Single-Photon-Decay spectroscopy by means of a Laplce Transform method: Application to a crystal of TMMC", Appl. Spect., 43, 324 (1989)

3. F. Moreno, R.J. López, F. González, "Analysis of multiexponential decays through a Sine Transform method in Single Photon decay spectroscopy", Proc. SPIE 1204, 206 (1990)

4. B.E.A. Saleh, "Photoelectron Statistics". Springer-Verlag, Berlin, 1978. Chap. 3, pp. 57-84.

5. H.P. Good, A.J. Kallir, U.P. Wild, "Optimum pulse repetition rates for single photon counting experiments", J. Lumin. 29, 491 (1984)

6. S.J. Yakowitz, "Computational Probability and Simulation", Addison-Wesley Pub. Co. (1977)

7. P.R. Bevington, "Data reduction and error analysis for the physical sciences", McGraw Hill, New York, 1969, Chap. 10 


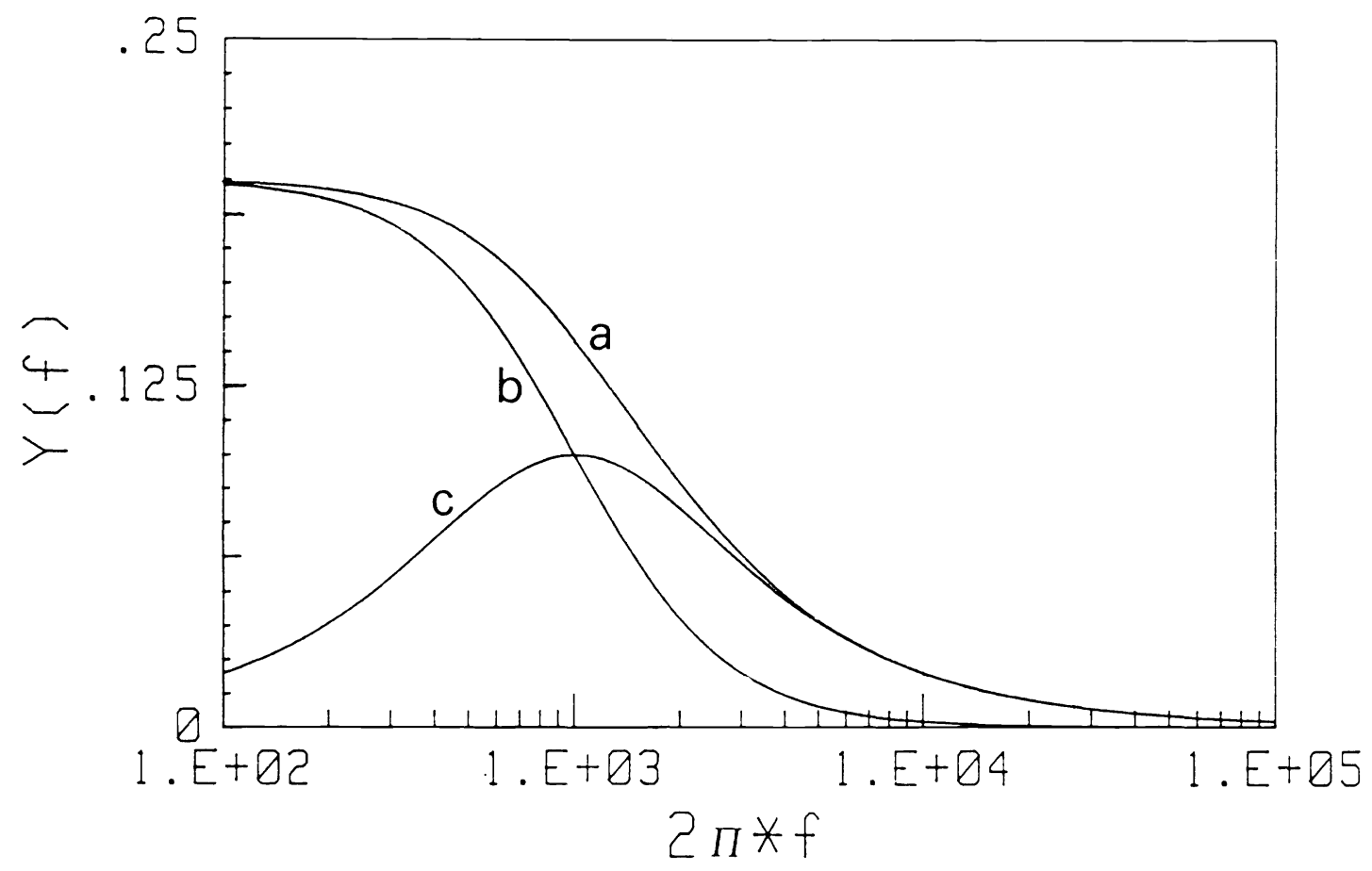

Figure 1.- Plot of : a) $\left.I T_{F}(f) \mid, b\right) T_{\cos }(f)$ and c) $T_{\sin }(f)$ versus $2 \pi f$. The values for $I_{0}$ and $\tau_{0}$ are 200 photoelectrons/sec. and $1 \mathrm{msec}$. respectively.

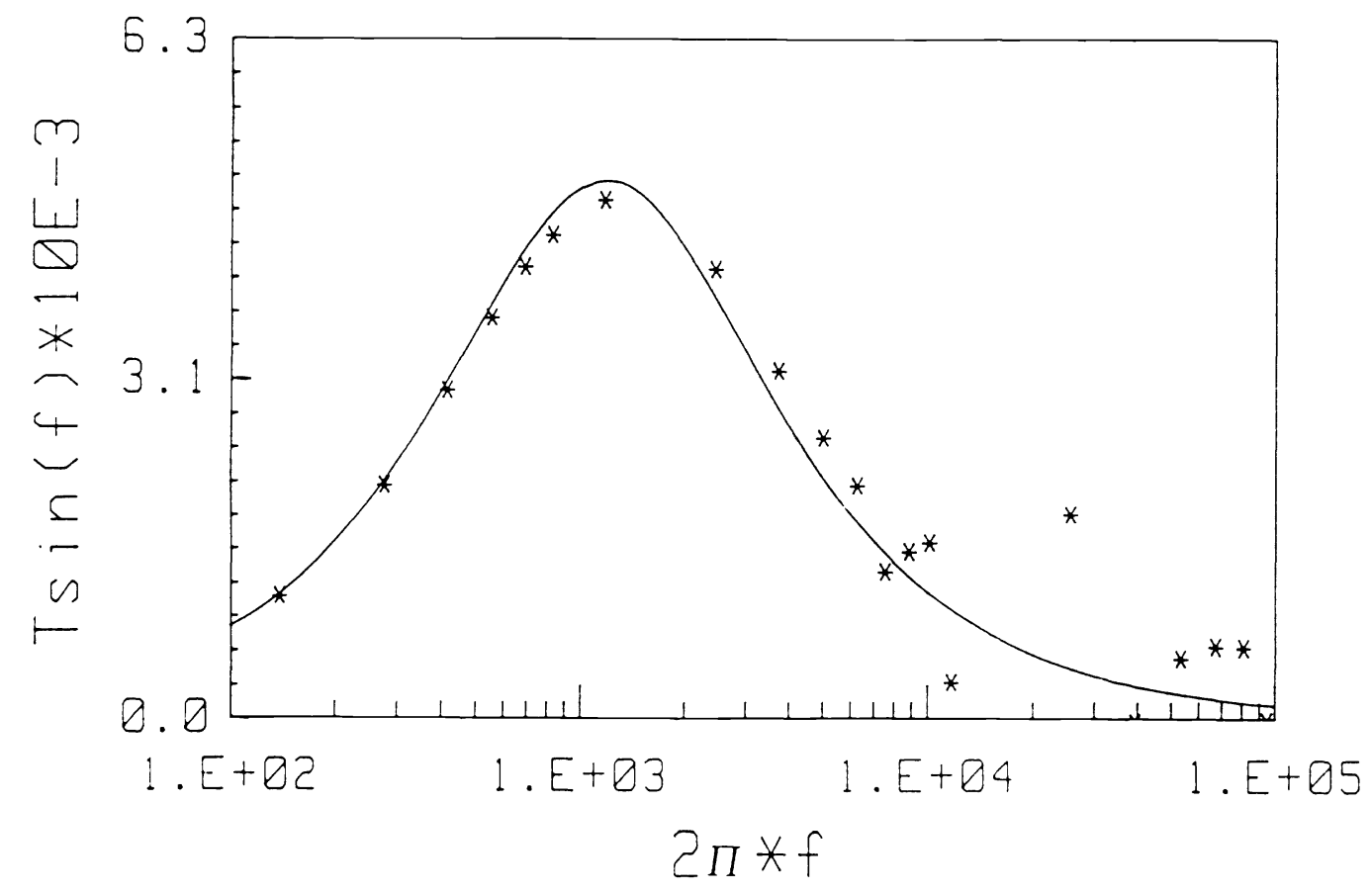

Figure 2.-Experimental simulation of $T_{\sin }(f)$.( The experimental values are represented by *) for $\alpha=0.01$ and $N_{c}=10^{4}$. 


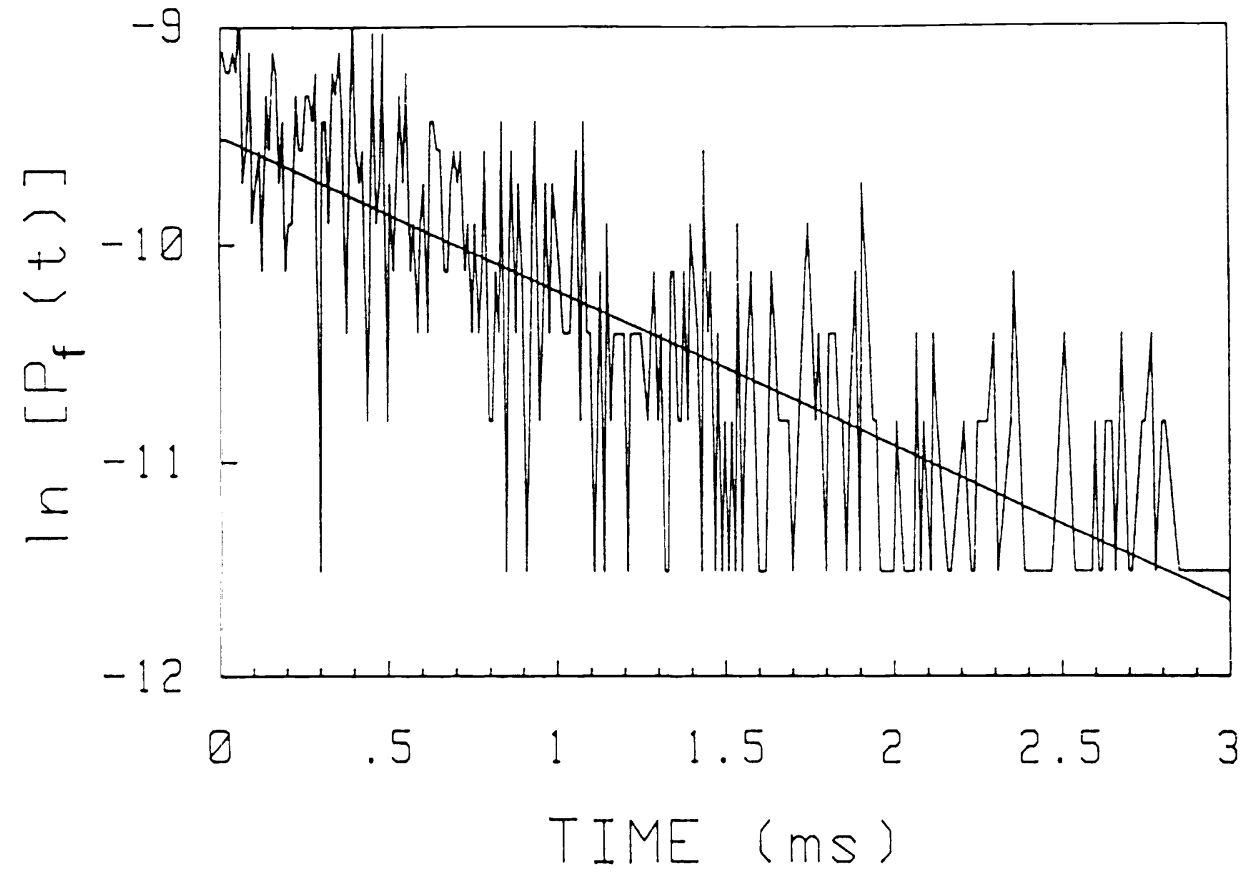

Figure 3.- Experimental simulation of $\mathrm{P}_{\mathrm{f}}(\mathrm{t})$ (logarithmic scale) obtained by means of a multichannel with 300 channels $\left(\alpha=0.01, \tau_{0}=1 \mathrm{msec}\right.$. and $\left.N_{c}=105\right)$. Continuous line represents the theoretical fitted function.

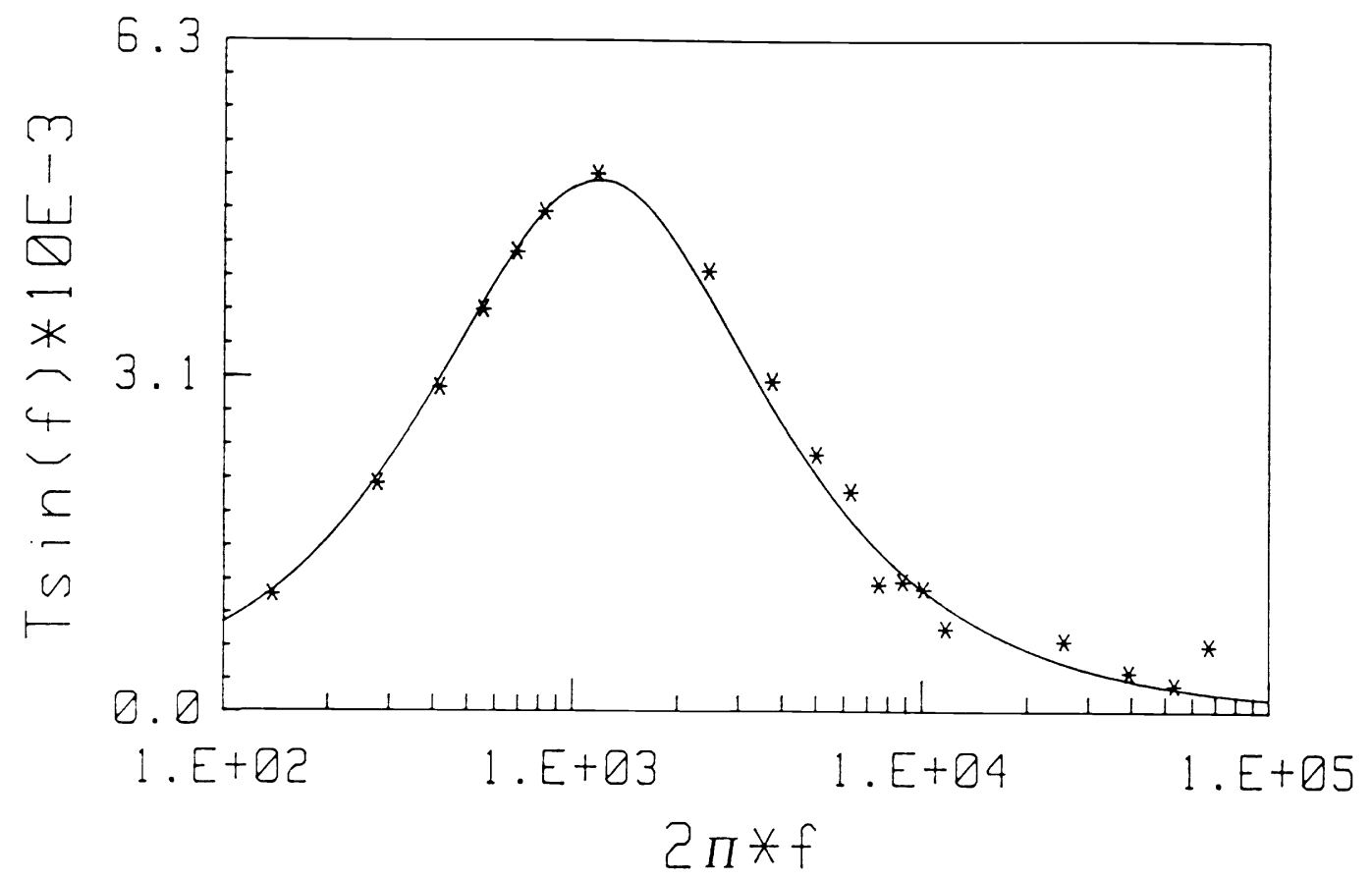

Figure 4.- Experimental simulation of $T_{\sin }(f)$ ( the experimental values are represented by ${ }^{*}$ ) for $\alpha=0.01, \tau_{0}=1 \mathrm{msec}$. and $\mathrm{N}_{\mathrm{c}}=105$. 


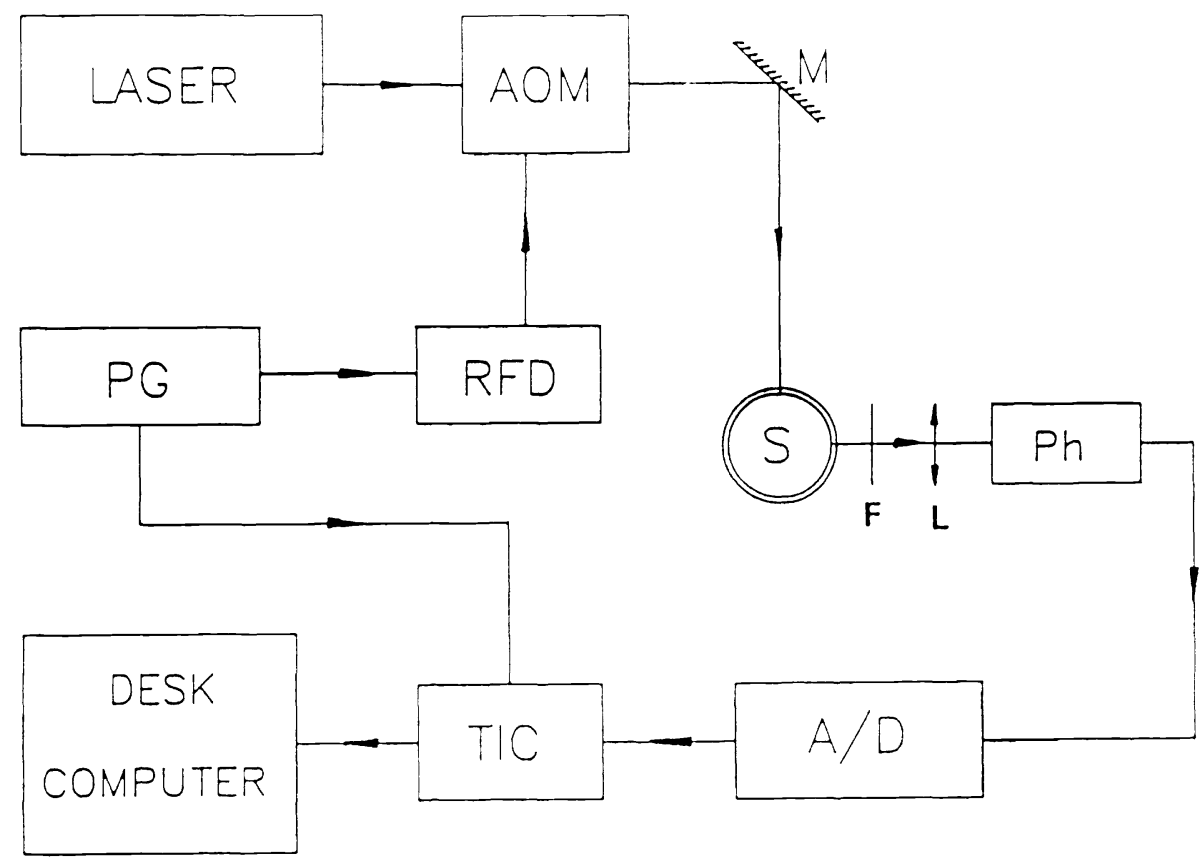

Figure 5.- Experimental setup. AOM :Acousto-optic light modulator; M: Mirror; Ph:photomultiplier; S:Sample; RFD:Radio-frequency driver; PG:Pulse generator; A/D:Amplifier-discriminator; TIC: Time-interval counter; F:Filter (650 nm); L:Lens.

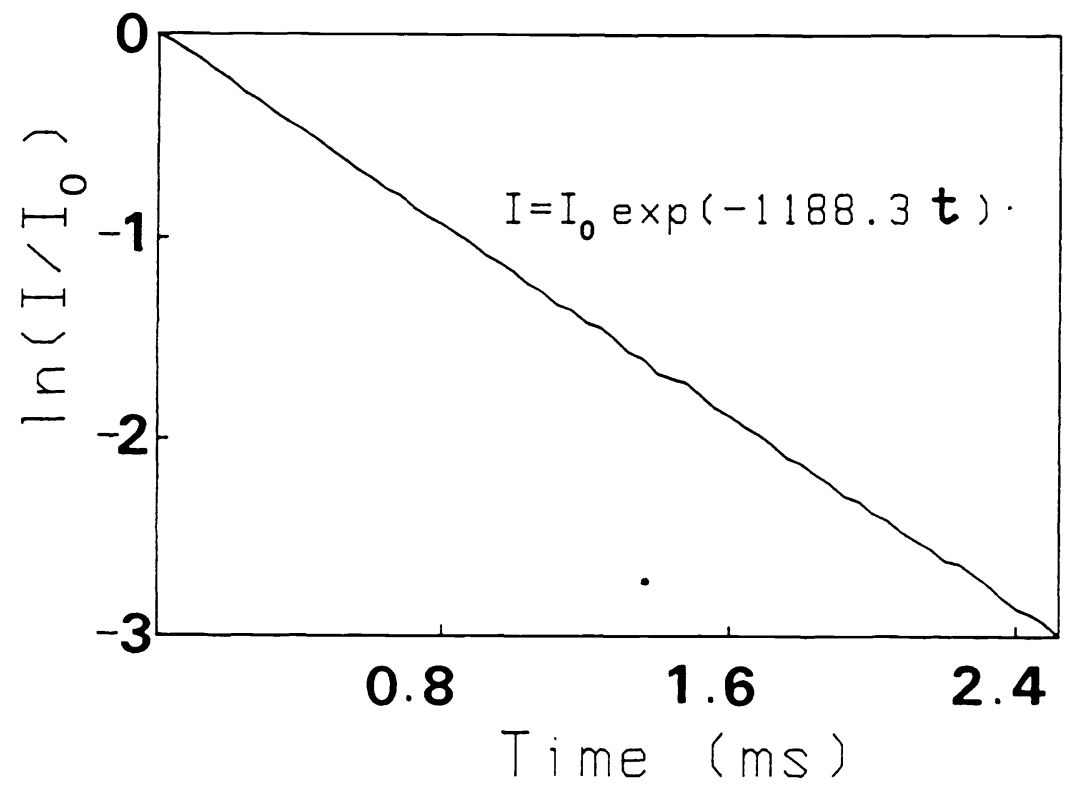

Figure 6.-Fluorescence time behaviour (logarithmic scale) of undopped sample of TMMC used in the experiment. 


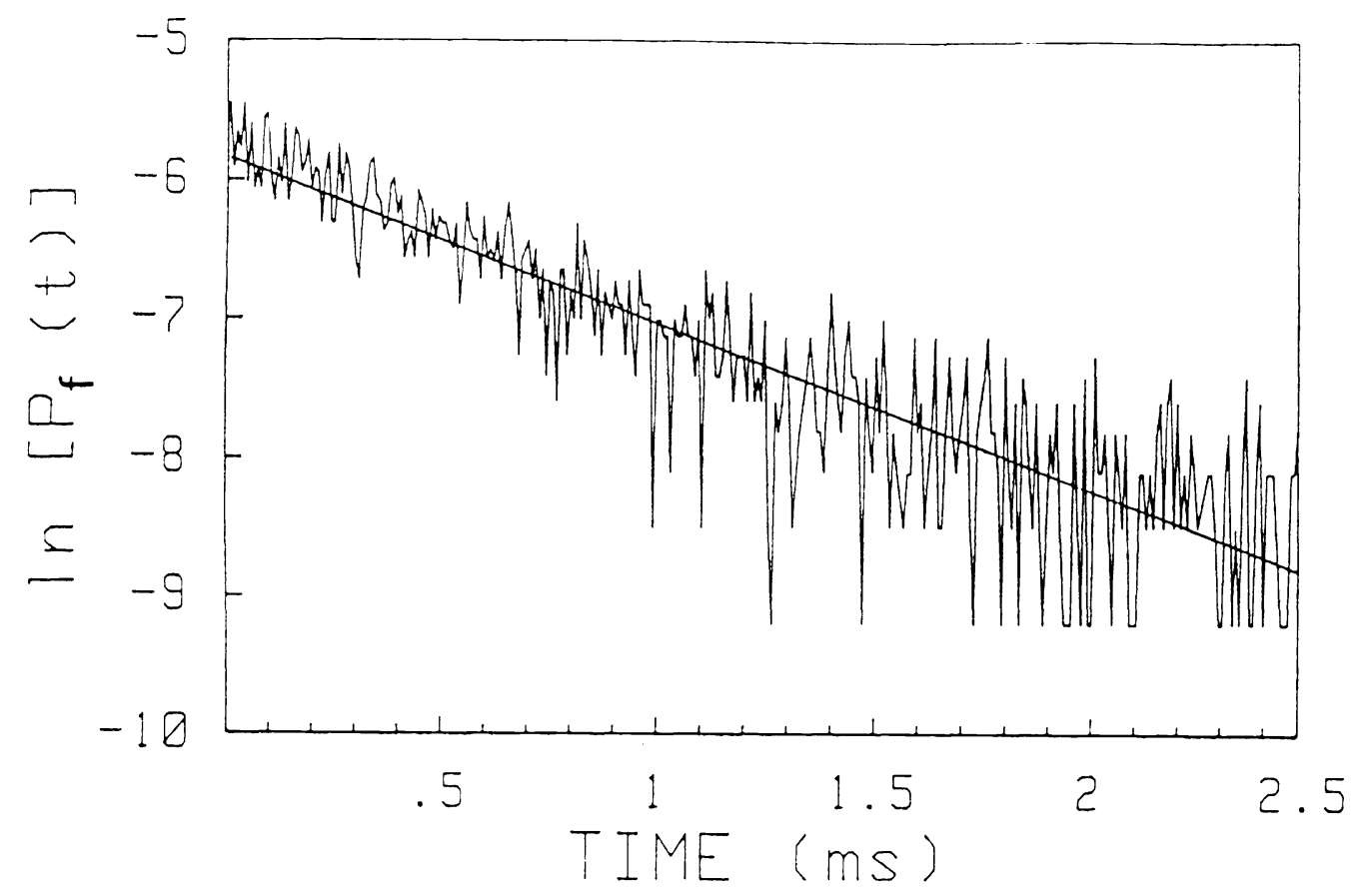

Figure 7.-Experimental result for $P_{f}(t)$ (logarithmic scale) obtained by means of a multichannel analyzer with 300 channels. Continuous line represents the theoretical fitted function.

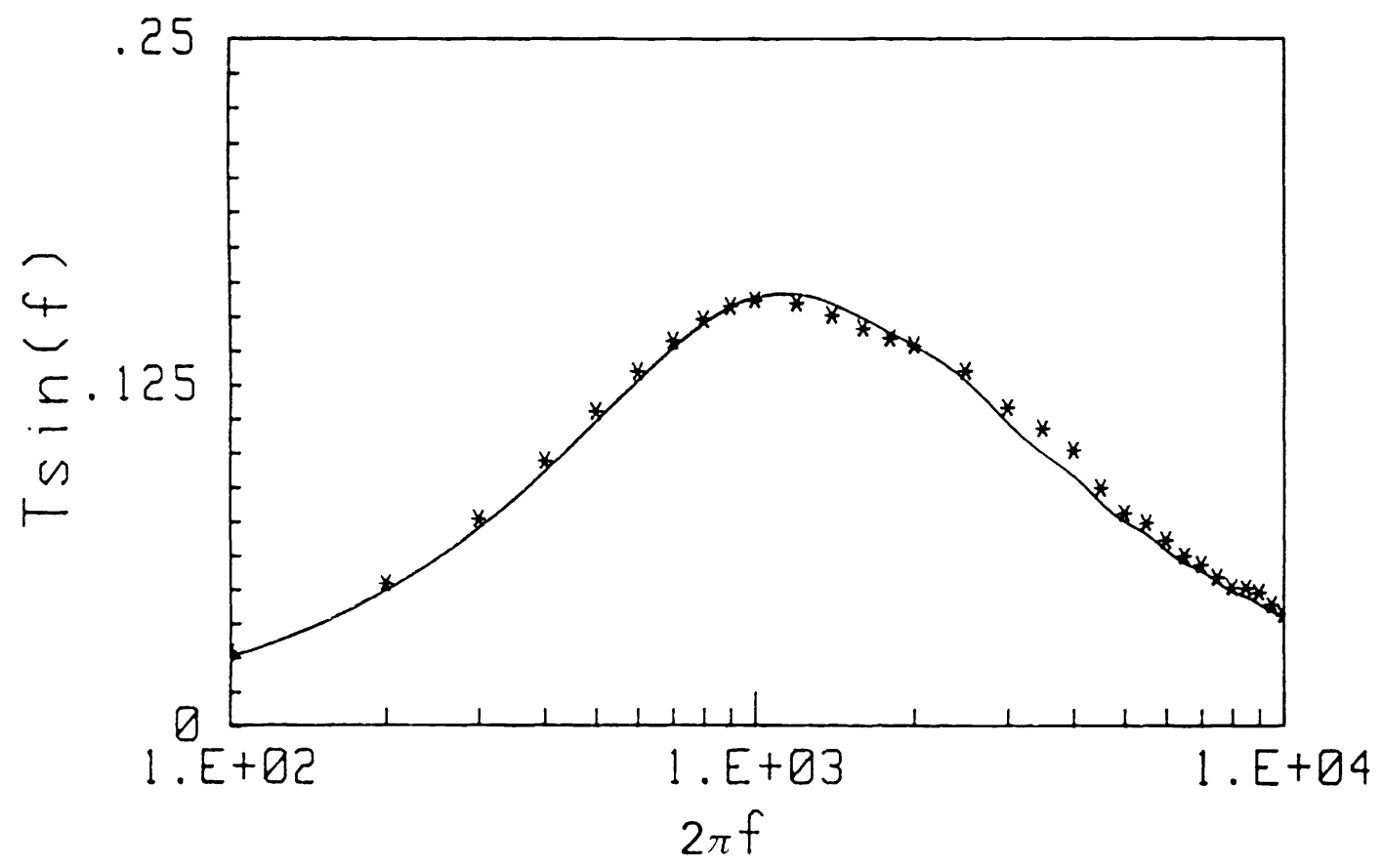

Figure 8.-Plot of one of the series measured for $T_{\sin }(f)(*)$ together with the theoretical fitted function (continuous line). 
Fitted function

\begin{tabular}{|c|c|c|}
\hline Parameter & $P_{f}(t)$ & $T_{\sin }(f)$ \\
\hline$\tau_{0}$ & $860.4 \mu \mathrm{s}$ & $818.5 \mu \mathrm{s}$ \\
\hline $\mathrm{e}_{\tau_{0}}(\%)$ & 3.7 & 2.2 \\
\hline$x_{v}^{2}$ & 62 & 0.8 \\
\hline $\mathrm{r}^{2}$ & 0.76 & --. \\
\hline
\end{tabular}

Table 1. Fitting results for $P_{f}(t)$ and $T_{\sin }(f) . \tau_{0}=$ mean value of $\tau_{0}, e_{\tau_{0}}=$ error in $\tau_{0}$ for ten measured series, $\chi_{v}{ }^{2}=$ value of the reduced chi-square parameter, $r=$ regression coefficient for the straight-line fitting. 